Check for updates

Cite this: J. Mater. Chem. A, 2022, 10 4355

Received 9th November 2021

Accepted 18th January 2022

DOI: $10.1039 / \mathrm{d} 1 \mathrm{ta0} 0620 \mathrm{f}$

rsc.li/materials-a

\section{Relevance of $\mathrm{Ge}$ incorporation to control the physical behaviour of point defects in kesterite $\uparrow$}

\author{
Thomas Ratz, (D)*ab Ngoc Duy Nguyen, (D) a Guy Brammertz, (D) ${ }^{c}$ Bart Vermang (D) bcd \\ and Jean-Yves Raty ${ }^{a}$
}

To reduce the prominent $V_{O C}$-deficit that limits kesterite-based solar cell efficiencies, $G e$ has been proposed over the recent years with encouraging results as the reduction of the non-radiative recombination rate is considered as an approach to improve the well-known Sn-kesterite world record efficiency. To gain further insight into this mechanism, we investigate the physical behaviour of intrinsic point defects upon both $\mathrm{Ge}$ doping and alloying of $\mathrm{Cu}_{2} \mathrm{ZnSnS}_{4}$ kesterite. Using a first-principles approach, we confirm the p-type conductivity of both $\mathrm{Cu}_{2} \mathrm{ZnSnS}_{4}$ and $\mathrm{Cu}_{2} \mathrm{ZnGeS}$, attributed to the low formation energies of the $\mathrm{V}_{\mathrm{Cu}}$ and $\mathrm{Cu}_{\mathrm{Zn}}$ acceptor defects within the whole stable phase diagram range. By doping of the Sn-kesterite matrix, we report the lowest formation energy for the substitutional defect $\mathrm{Ge}_{\mathrm{Sn}}$. We also confirm the detrimental role of the substitutional defects $X_{\mathrm{Zn}}(X=\mathrm{Sn}, \mathrm{Ge})$ acting as recombination centres within the $\mathrm{Sn}$-based, the Ge-doped and the Ge-based kesterite. Upon Ge incorporation, we highlight, along with the increase of the $X_{Z n}(X=S n, G e)$ neutral defect formation energy, the reduction of the lattice distortion resulting in the reduction of the carrier capture cross section. Both of these elements lead to a decrease of the non-radiative recombination rate within the bulk material following Sn substitution by Ge.

\section{Introduction}

Emerging solar cell technologies often struggle with a minority carrier lifetime, leading to large open circuit voltage deficit $\left(V_{\mathrm{OC}^{-}}\right.$ deficit) and consequently low solar cell efficiency. With no exception, in opposition to their well-established $\mathrm{CuInGa}(\mathrm{S}, \mathrm{Se})_{2}$ chalcogenide parent showing high cell efficiency, ${ }^{1,2}$ kesterite materials are faced with efficiency issues..$^{3-5} \mathrm{As}$-possible culprits of the actual limitation, electronic defects acting as recombination centres have been pointed out ${ }^{6-11}$ together with other obstacles such as band alignments, ${ }^{9,12,13}$ secondary phases ${ }^{10,11}$

${ }^{a} C E S A M|Q-M A T|$ Solid State Physics, Interfaces and Nanostructures, University of Liège, Allée du Six Août 19, B-4000 Liège, Belgium

${ }^{b}$ Institute for Material Research (IMO), Hasselt University, Agoralaan gebouw $\mathrm{H}, \mathrm{B}$ 3590 Diepenbeek, Belgium

'IMEC Division IMOMEC | Partner in Solliance, Wetenschapspark 1, B-3590 Diepenbeek, Belgium

${ }^{d}$ Energyville, Thor Park 8320, B-3600 Genk, Belgium

$\dagger$ Electronic supplementary information (ESI) available: Further details about the theoretical framework used to compute the formation energy of point defects, the phase diagrams, the Fermi energy level under thermodynamic equilibrium conditions and the formation energy correction terms are provided. In addition, the specific formation energy values of the kesterite materials, secondary phases and the chemical potential values of the points labelled in the phase diagrams are provided as well as the kesterite lattice parameters. Relaxed structures (CONTCAR) and raw calculation outputs (WAVECAR, CHGCAR,etc.) as well as the resulting calculation total energies are available upon reasonable request to the corresponding author. See DOI: 10.1039/d1ta09620f and/or band tailing caused by electrostatic potential fluctuations due to the presence of charged electronic defects. ${ }^{\mathbf{8}, 9,14}$

Focusing on defects, as a result of the complex structure of kesterite materials, a wide range of intrinsic and cluster defects can form within the crystal, leading to various impacts on the kesterite absorber layer opto-electronic properties. Using firstprinciples calculations, Chen et al. were able to predict the p-type conductivity of $\mathrm{Cu}_{2} \mathrm{ZnSn}(\mathrm{S}, \mathrm{Se})_{4}$ via the high population of the $\mathrm{Cu}_{\mathrm{Zn}}$ and $\mathrm{V}_{\mathrm{Cu}}$ defects while identifying the $\left[2 \mathrm{Cu}_{\mathrm{Zn}}+\mathrm{Sn}_{\mathrm{Zn}}\right]$ cluster defect as a recombination centre leading to charge carrier losses. ${ }^{6}$ Experimentally, Dimitrievska et al. reported the possible tuning of the $V_{\mathrm{OC}}$ value according to the $\mathrm{Cu}$ concentration and consequently the amount of $\left[\mathrm{V}_{\mathrm{Cu}}+\mathrm{Zn}_{\mathrm{Cu}}\right]$ defect clusters. ${ }^{10}$ More recently, Kim et al. identified the intrinsic defect $\mathrm{Sn}_{\mathrm{Zn}}$ as the origin of the electron capture and emission in the $\mathrm{Cu}_{2} \mathrm{ZnSnS}_{4}$ compound resulting of the Sn multivalence. ${ }^{7,15}$ The same authors also highlighted the large lattice distortion attributed to the $\mathrm{Sn}$ in its oxidation state +2 with respect to its +4 state leading to a large carrier capture cross section associated with this so-called "lonepair effect". ${ }^{16}$ Furthermore, Gong et al. established a link between the $\mathrm{Sn}$ oxidation states $\mathrm{Sn}^{2+}$ and $\mathrm{Sn}^{4+}$ and the kesterite growth conditions. ${ }^{17}$ Consequently, gaining further knowledge on point defects and cluster defects in kesterite materials could allow the control of the absorber layer physical properties in view of increasing kesterite-based cell efficiencies.

Over the recent years, attempts have been made to circumvent these actual limitations using alloying and doping of 
kesterite materials with other elements., ${ }^{\mathbf{3} \mathbf{1 8 - 2 3}}$ Both theoretical and experimental approaches have been used. A wide range of cationic substitutions have been investigated: $\mathrm{Cu}$ by $\mathrm{Ag},{ }^{\mathbf{2 4 , 2 5}} \mathrm{Zn}$ by $\mathrm{Cd},{ }^{24,26-28} \mathrm{Sn}$ by $\mathrm{Ge},{ }^{28-32} \mathrm{~S}$ by $\mathrm{Se}^{4}$ and doping of both $\mathrm{Cu}_{2^{-}}$ $\mathrm{ZnSnS}_{4}$ and $\mathrm{Cu}_{2} \mathrm{ZnSnSe}_{4}$ by $\mathrm{Na}, \mathrm{Li}, \mathrm{Ga}^{33}$ and $\mathrm{Ge}^{\mathbf{1 8 , 3 4 - 3 6}}$ or even using more exotic elements as in ref. ${ }^{20}$. Some of these substitutions resulted in cell efficiencies as large as $12.3 \%$ as in the case of Ga or Ge doping. ${ }^{33,34}$ Double cation incorporation like Ge and $\mathrm{Ag}^{37}$ or $\mathrm{Ge}$ and $\mathrm{Cd}^{28,38}$ was also realised simultaneously combining the benefits of both substitutional elements. Nevertheless, the reported efficiencies are still below the worldrecord of pure Sn-kesterite compounds. ${ }^{39}$

From these investigations, Ge emerged as an interesting doping/alloying element as several studies reported high solar cell efficiencies through the improvement of the $V_{\mathrm{OC}}$ values following the incorporation of small amounts of $\mathrm{Ge}^{35}$ or via the complete substitution of $\mathrm{Sn}$ by $\mathrm{Ge} .{ }^{31}$ In a recent study, Deng et al. demonstrated experimentally that $\mathrm{Ge}^{4+}$ can be introduced in $\mathrm{Cu}_{2} \mathrm{ZnSnS}_{4}$ to suppress the detrimental deep $\mathrm{Sn}_{\mathrm{Zn}}$ defects. ${ }^{36}$ In addition, compared to $\mathrm{Cu}_{2} \mathrm{ZnSnS}_{4}$, pure Ge-kesterite absorber layers present a larger band gap value which limits the maximal single solar cell efficiency. ${ }^{40}$ Nevertheless, Ge alloying could be used for the synthesis of wide band gap kesterite which is of high interest for top cells in a tandem approach. ${ }^{29,30}$ Gaining further insight into the physical behaviour of intrinsic defects in Ge-kesterite and into the mechanisms of Ge doping within the Sn-kesterite is therefore strongly desirable.

Moreover, although several material modelling studies have focused on the physical behaviour of defects in kesterite compounds, ${ }^{6,7,20,33}$ only a few studies have been dedicated so far to Ge compounds. ${ }^{\mathbf{2 8 , 4 1 , 4 2}}$ To fill this void, in this work, we report the investigation of point defects in both $\mathrm{Cu}_{2} \mathrm{ZnSnS}_{4}$ and $\mathrm{Cu}_{2}$ $\mathrm{ZnGeS}_{4}$. We first study the physical behaviour of intrinsic point defects in both kesterite materials to highlight the impact of Ge alloying and secondly, we investigate Ge-related point defects in the Sn-kesterite compound to illustrate the Ge doping mechanism. Focusing on the physical behaviour of point defects in these materials, our aim is to establish a link between the growth conditions of the kesterite thin films, the formation of point defects and the resulting kesterite solar cell performances by identifying the defect physical behaviour (dopant or recombination centre). In addition, based on the empirical rule proposed by Li et al. we derive meaningful trends concerning the defect carrier capture cross sections. Indeed, it was reported that this quantity can be related to lattice distortion caused by the defect incorporation in its various charge states. ${ }^{43}$

The paper is organised as follows. The phase diagrams of the two kesterites are first presented. Once appropriate chemical potential ranges are set, avoiding secondary phases and obtaining the desired kesterite phase, the defect formation energies are obtained according to the Fermi level position. Then, we present the defect charged states and their possible ionisation levels in order to evaluate their physical behaviour in their various electronic configurations. This approach allows us to identify their roles as a dopant or as a recombination centre. Finally, in the last part of this paper, we present a study of the lattice distortions around the different incorporated defects. This information allows us to provide a guide for the extraction of general trends concerning the capture cross sections of the various point defects at play. As a result, we are able to predict which point defects are the most abundant in the kesterite materials and eventually characterise the defect levels (acceptor, donor or recombination centre) and their relative impact on the capture of charge carriers. Considering various locations in the phase diagram, we also consider different growth conditions of the kesterite materials. This study allows us to highlight (i) the impact of Ge-alloying of kesterite on the intrinsic point defect physical behaviour and (ii) the physical behaviour of Ge dopants in $\mathrm{Cu}_{2} \mathrm{ZnSnS}_{4}$.

\section{Computational methodology}

\subsection{Theoretical framework}

Beyond materials properties predictions, the first-principles approach is a powerful tool to understand the behaviour of defects in semiconductor compounds. ${ }^{\mathbf{4 4 - 4 7}}$ In this work, the supercell approach is considered and the calculations are performed with a 64-atom supercell corresponding to an expansion of $2 \times 2 \times 2$ of the kesterite conventional cell as presented in Fig. 1.

Using this approach, the formation energy of a defect $\alpha$ in a charge state $q$ can be calculated as follows,

$$
\begin{aligned}
\Delta H_{\mathrm{F}}\left(\alpha, q, E_{\mathrm{F}}, \mu_{i}\right)= & E(\alpha, q)-E_{\mathrm{host}}-\sum_{i} n_{i}\left(E_{i}+\mu_{i}\right) \\
& +q\left[\varepsilon_{\mathrm{VBM}, \text { host }}+E_{\mathrm{F}}\right],
\end{aligned}
$$

where $E(\alpha, q)$ is the total energy of the supercell with a defect $\alpha$ in the charge state $q ; E_{\text {host }}$ is the total energy of the 64-atom pristine supercell. $n_{i}$ is the number of atom(s) of the species $i$ removed $(<0)$ from or added $(>0)$ to the host supercell with $E_{i}$ being the energy per atom of the pure phase of the species $i$ and $\mu_{i}$ the chemical potential of the corresponding element. A variation in the synthesis conditions can change the thin film

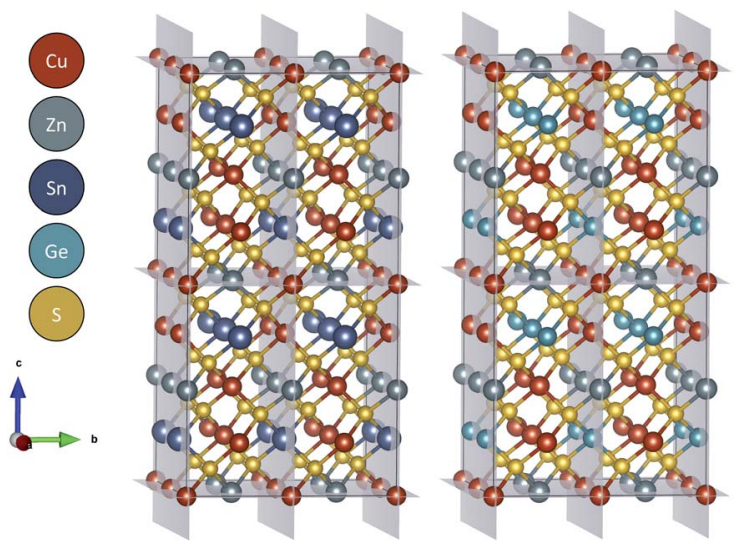

Fig. 1 Pristine kesterite 64-atom supercells used to compute the defect formation energies. Each supercell corresponds to an expansion of $2 \times 2 \times 2$ of the kesterite conventional cell as represented by the grey shadings. 
composition and, consequently, the environment in which the defect will be formed. As a result, the point defect formation energy and the defect concentration will be impacted according the amount of energy required by the exchange of particles necessary to form the defect. This energy cost is described by the chemical potential of the chemical species $\left(\mu_{i}\right)$ which is defined as the Gibbs free energy variation caused by the exchange of particles between the system and an external reservoir, $\mu_{i}=\left(\frac{\mathrm{d} G}{\mathrm{~d} N_{i}}\right) \cdot{ }^{44}$ To obtain the chemical potential values $\mu_{i}$ leading to the formation of a stable kesterite phase without secondary phases, the kesterite phase diagrams have first to be computed. To do so, a set of thermodynamic conditions must be fulfilled as presented in the ESI. $\uparrow$ Then, assuming a defect with a charge state $q$, a fourth term is added to eqn (1). In this term, $\varepsilon_{\mathrm{VBM}}$,host refers to the valence band maximum (VBM) of the host supercell and $E_{\mathrm{F}}$ is the Fermi level acting as a parameter of the defect formation energy function and ranging from the VBM to the band gap energy $E_{\mathrm{G}}$ of the kesterite material. Furthermore, based on the formation energy of a given defect, the position of its ionisation levels in the materials band gap can be obtained using the following relationship:

$$
\varepsilon\left(\alpha, q, q^{\prime}\right)=\frac{\Delta H_{\mathrm{F}}(\alpha, q)-\Delta H_{\mathrm{F}}\left(\alpha, q^{\prime}\right)}{q^{\prime}-q},
$$

with $q, q^{\prime}$ being the charge states of the defect $\alpha$ considered for the transition.

\subsection{Methodology}

To compute the total energies of the defected and host supercells (as required in eqn (1)) and of the secondary phases and pure elemental phases (see ESI $\dagger$ ), first-principles calculations have been performed using the Vienna $A b$ initio Simulation Package (VASP) $\operatorname{code}^{48}$ with the ProjectorAugmented Wave (PAW) potential method. ${ }^{49}$ Perdew-BurkeErnzerhof (PBE) GGA pseudo-potentials ${ }^{50}$ were used with the following valence electrons considered for each element: $\mathrm{Cu}$ : $3 \mathrm{~d}^{10} 4 \mathrm{~s}^{1}$, Zn: $3 \mathrm{~d}^{10} 4 \mathrm{~s}^{2}$, Sn: $4 \mathrm{~d}^{10} 5 \mathrm{~s}^{2} 5 \mathrm{p}^{2}$, Ge: $3 \mathrm{~d}^{10} 4 \mathrm{~s}^{2} 4 \mathrm{p}^{2}$ and $\mathrm{S}$ : $3 \mathrm{~s}^{2} 3 \mathrm{p}^{4}$. Based on the previously presented supercell approach, within a 64-atom supercell, ionic and electronic relaxations were achieved using a cut-off energy of $520 \mathrm{eV}$ and a $\Gamma$-centered uniform $k$-points mesh of $2 \times 2 \times 2 k$-points. Applying the strongly constrained and appropriately normed semilocal density functional (SCAN), ${ }^{\mathbf{5 1 , 5 2}}$ the structures were relaxed until the numerical convergence regarding the self-consistent cycles reached forces between ions less than $10^{-3} \mathrm{eV} \AA^{-1}$. Starting from the relaxed structures, a single-shot calculation using the Heyd-Scuseria-Ernzerhof exchange-correlation functional (HSE06) ${ }^{53}$ is performed using an energy convergence criterion upon $10^{-3} \mathrm{eV}$. The combination of SCAN ionic relaxations followed by a single HSE06 electronic relaxation was reported as an efficient method to obtain accurate results by Fritsch et al. in ref. ${ }^{54}$. Concerning Ge-containing compounds, a correction was applied to the Ge-chalcogenide formation energies to tackle the systematic underestimation as reported by Wexler et $a l^{42}$ (see the ESI $\dagger$ ).

\section{Results and discussion}

\subsection{Kesterite growth: chemical environment}

Let us first determine the chemical environment in which the kesterite materials can be synthesised without secondary phases. In Fig. 2, the phase diagrams of Sn-based and Ge-based kesterites are presented for three different $\mathrm{Cu}$ concentrations: $\mu_{\mathrm{Cu}}=-0.27$ (Cu-rich) eV, $\mu_{\mathrm{Cu}}=-0.55 \mathrm{eV}$ (Cu-moderate) and $\mu_{\mathrm{Cu}}=-0.82 \mathrm{eV}$ (Cu-poor).

A first general observation is the narrow chemical potential range in which the kesterite phase can be found without any secondary phase (green shading) in comparison to the possible chemical potential combinations that could lead to the formation of the kesterite (grey shading). For lower $\mathrm{Cu}$ concentrations, corresponding to a $\mu_{\mathrm{Cu}}$ value lower than $-0.82 \mathrm{eV}$ (resp. $-0.85 \mathrm{eV}$ for $\mathrm{Cu}_{2} \mathrm{ZnGeS}_{4}$ ), no pure kesterite phase is available within stable combinations of chemical potentials values. This means that below such a critical $\mu_{\mathrm{Cu}}$ value, the kesterite phase cannot be formed without the presence of secondary phases. In addition, from Fig. $2 \mathrm{a}-\mathrm{c}$, we observe a reduction of the stable kesterite phase area (grey shading). This reduction occurs as we move towards lower $\mathrm{Cu}$ concentrations (see the ESI $\dagger$ ). If one chemical potential absolute value increases for a constant kesterite formation energy, the available chemical potential combination range is reduced (i.e. the edge of the stable kesterite phase corresponding to $\mu_{\mathrm{S}}=0$ is shrunk). Physically, it means that for a lower chemical potential value corresponding to a lower concentration, another element concentration has to increase to keep obtaining a stable kesterite phase.

The reduction of the stable kesterite areas (grey shading) to pure kesterite areas (green shading) is the result of the multiple secondary phases that can be formed using the 4 chemical elements present in the kesterite compounds. As shown in Fig. 2a, around the green area, the dominant secondary phases are $\mathrm{ZnS}, \mathrm{Cu}_{7} \mathrm{~S}_{4}, \mathrm{Cu}_{2} \mathrm{SnS}_{3}$ and $\mathrm{SnS}$. The phases predicted by our calculations are in good agreement with those reported by Chen et al. in ref. ${ }^{6}$. In addition, in the recent review of Schorr et al., the authors reported the presence of $\mathrm{ZnS}$ in each thin film where the composition $\mathrm{Zn} / \mathrm{Sn}$ exceeded the value of 1 . It was also reported that the concentrations of both $\mathrm{ZnS}$ and $\mathrm{SnS}$ secondary phases increase upon deviation from the stoichiometry. ${ }^{10,11,55}$ Similarly, for the Ge-compound in Fig. 2d, the secondary phases located next to the pure kesterite phase are $\mathrm{ZnS}, \mathrm{Cu}_{7} \mathrm{~S}_{4}$ and $\mathrm{Cu}_{2} \mathrm{GeS}_{3}$. Experimentally, using XRD and HAADF-STEM imaging, Khelifi et al. reported that the ZnSe secondary phase at the top of the absorber layer and a thick $\mathrm{Cu}_{2} \mathrm{GeSe}_{3}$ secondary phase (120-160 nm) at the bottom of the kesterite thin film were responsible for the main efficiency loss. ${ }^{29}$ In the case of the Ge-based compound and in comparison with the SnS phase in the Sn-kesterite, the GeS secondary phase appears to be shifted towards positive Ge chemical potential values. As a result, the Ge-kesterite pure phase area (green shading) is slightly larger compared to the Sn-kesterite one. Following the phase diagram evolution from Fig. 2a-c, we observe that, as the absolute value of the $\mathrm{Cu}$ chemical potential increases, the $\mathrm{SnS}_{2}$ and $\mathrm{SnS}$ secondary phase limits shift 


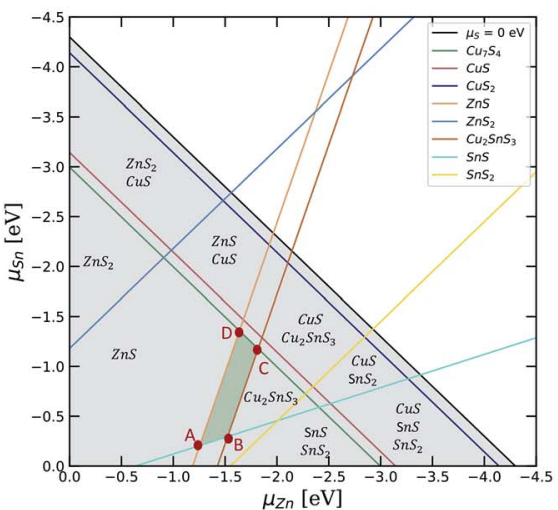

(a) $\mathrm{Cu}_{2} \mathrm{ZnSnS}_{4}-\mu_{\mathrm{Cu}}=-0.27 \mathrm{eV}$

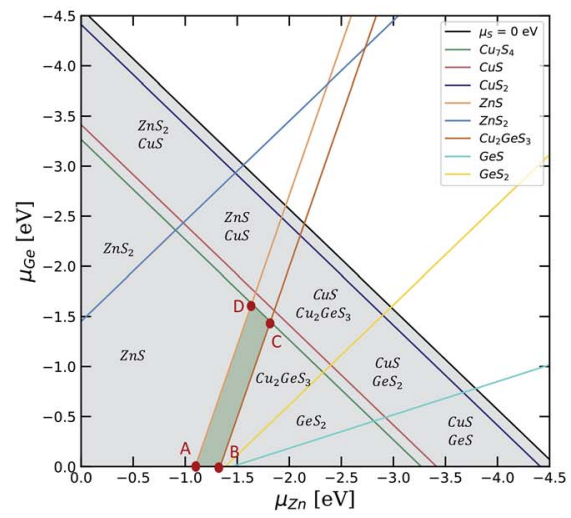

(d) $\mathrm{Cu}_{2} \mathrm{ZnGeS}_{4}-\mu_{\mathrm{Cu}}=-0.27 \mathrm{eV}$

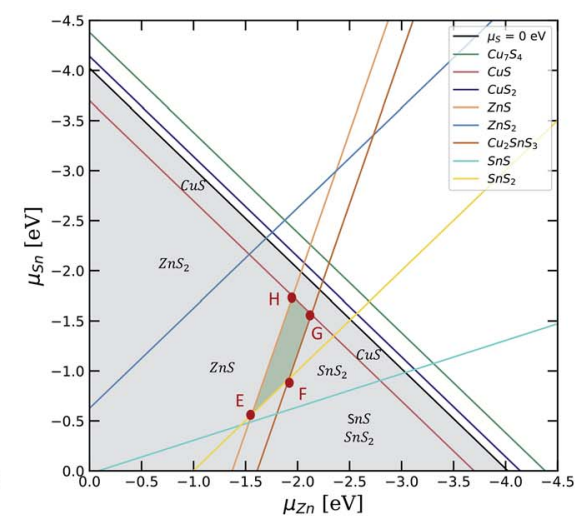

(b) $\mathrm{Cu}_{2} \mathrm{ZnSnS}_{4}-\mu_{\mathrm{Cu}}=-0.55 \mathrm{eV}$

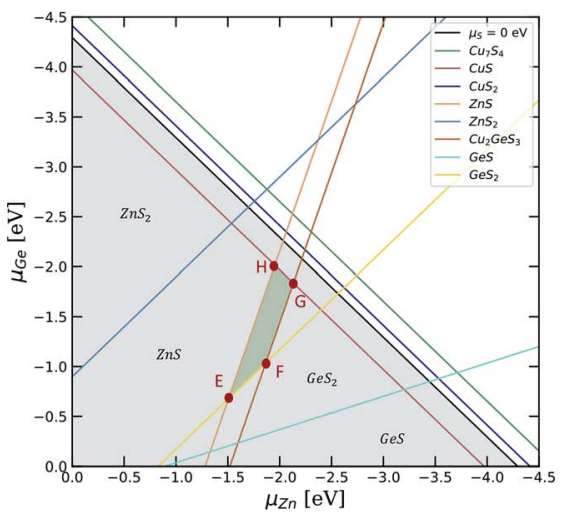

(e) $\mathrm{Cu}_{2} \mathrm{ZnGeS}_{4}-\mu_{\mathrm{Cu}}=-0.55 \mathrm{eV}$

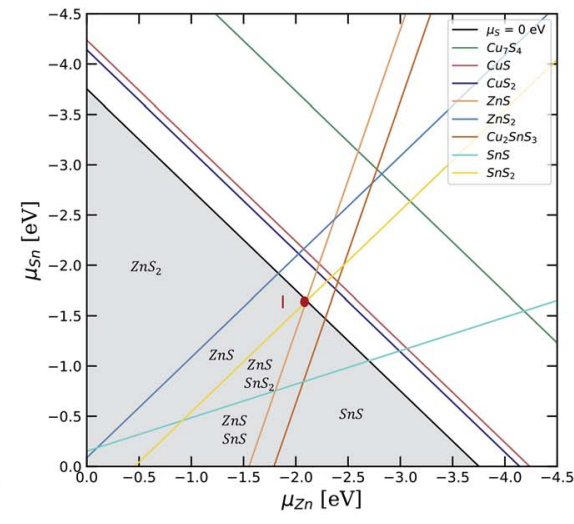

(c) $\mathrm{Cu}_{2} \mathrm{ZnSnS}_{4}-\mu_{\mathrm{Cu}}=-0.82 \mathrm{eV}$

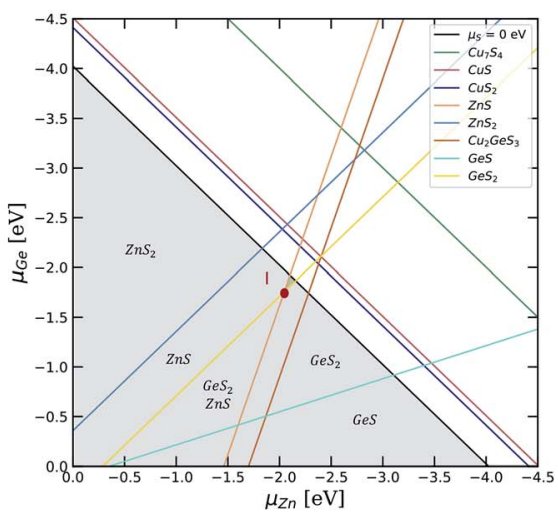

(f) $\mathrm{Cu}_{2} \mathrm{ZnGeS}_{4}-\mu_{\mathrm{Cu}}=-0.82 \mathrm{eV}$

Fig. 2 Phase diagrams of $\mathrm{Cu}_{2} \mathrm{ZnSnS}_{4}(\mathrm{a}-\mathrm{c})$ and $\mathrm{Cu}_{2} \mathrm{ZnGeS}_{4}(\mathrm{~d}-\mathrm{f})$ for three different $\mathrm{Cu}$ chemical potentials. Each line corresponds to a secondary phase as listed in the legends (see the ESI† for secondary phases calculations). The grey-shaded areas correspond to chemical potential values for which the kesterite can be thermodynamically synthesised (stable phase). Upon taking into account the possible presence of the secondary phases, the green shading indicates the chemical potential ranges for which a kesterite stable phase is encountered without any secondary phase.

towards lower Sn concentrations while the $\mathrm{ZnS}$ secondary edge shifts towards lower $\mathrm{Zn}$ concentrations until both $\mathrm{SnS}_{2}$ and $\mathrm{ZnS}$ secondary phase edges cross each other for a $\mathrm{Cu}$ chemical potential value of $\mu_{\mathrm{Cu}}=-0.82 \mathrm{eV}$, leading to the vanishing of the pure kesterite area. The same behaviour can be observed for $\mathrm{Cu}_{2} \mathrm{ZnGeS}_{4}$ with an extreme copper chemical potential value of $-0.85 \mathrm{eV}$ involving the following secondary phases: $\mathrm{GeS}, \mathrm{GeS}_{2}$ and $\mathrm{ZnS}$. Recently, Choubrac et al. reported the effectiveness of a set of different surface treatments to get rid off all detrimental secondary phases for $\mathrm{Cu}_{2} \mathrm{ZnGeS}_{4}{ }^{31}$

Then, to compute Ge-doping point defects in the Sn-based kesterite, one has to obtain a chemical potential value for the Ge $\left(\mu_{\mathrm{Ge}}\right)$. To do so, as presented in the ESI, $\uparrow$ several additional secondary phases were computed.

In the next section, we will focus on the formation energy of the point defects using chemical potential combinations providing a pure kesterite phase. As explained with additional details in the ESI, $\dagger$ using eqn (1) to compute the defect formation energies, one has to ensure that the chemical reservoirs used (i.e. chemical potentials) contribute only to the formation of the kesterite phase and not to the synthesis of secondary phases.

\subsection{Intrinsic point defect formation energies}

In Fig. 3, we present the formation energies of kesterite point defects for the chemical potentials $\mu_{i}$ corresponding to point $\mathrm{E}$ in Fig. $2 b$ and e (see eqn (1)). The choice for this particular composition point was motivated by the perspective of selecting a chemical potential combination corresponding to a pure kesterite phase and as close as possible to the $\mathrm{Cu}$-poor and $\mathrm{Zn}$ rich conditions usually used to synthetise kesterite thin films. ${ }^{6,11,56}$ One has to keep in mind that Fig. 3 shows a glimpse of the defect formation energy function for one material growth condition. According to eqn (1), the defect formation energy $\Delta H_{\mathrm{F}}\left(E_{\mathrm{F}}\right)$ is represented as a function of the Fermi energy level within the kesterite band gaps as predicted by the HOMOLUMO Kohn-Sham eigenvalues extracted from the first-principles calculations which are equal to $1.32 \mathrm{eV}$ and $1.89 \mathrm{eV}$ respectively for $\mathrm{Cu}_{2} \mathrm{ZnSnS}_{4}$ and $\mathrm{Cu}_{2} \mathrm{ZnGeS}_{4}{ }^{{ }^{\mathbf{4 0}}}$

Let us first focus on the intrinsic point defect formation energies in the Sn-compound as it is used as our reference material. As shown in Fig. 3a, independent of the Fermi energy value, a first general trend is the lower formation energies of the vacancies (except $V_{\mathrm{Zn}}$ ) and the substitutional defects in comparison to the interstitial ones. This observation can be 

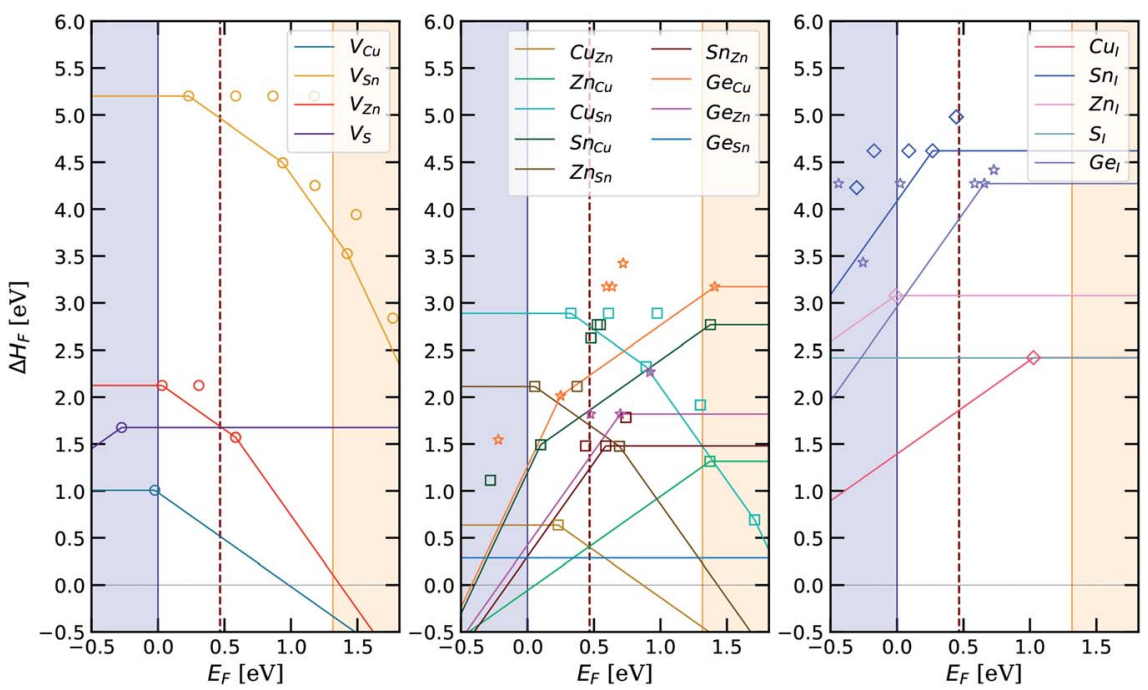

(a) $\mathrm{Cu}_{2} \mathrm{ZnSnS}_{4}$
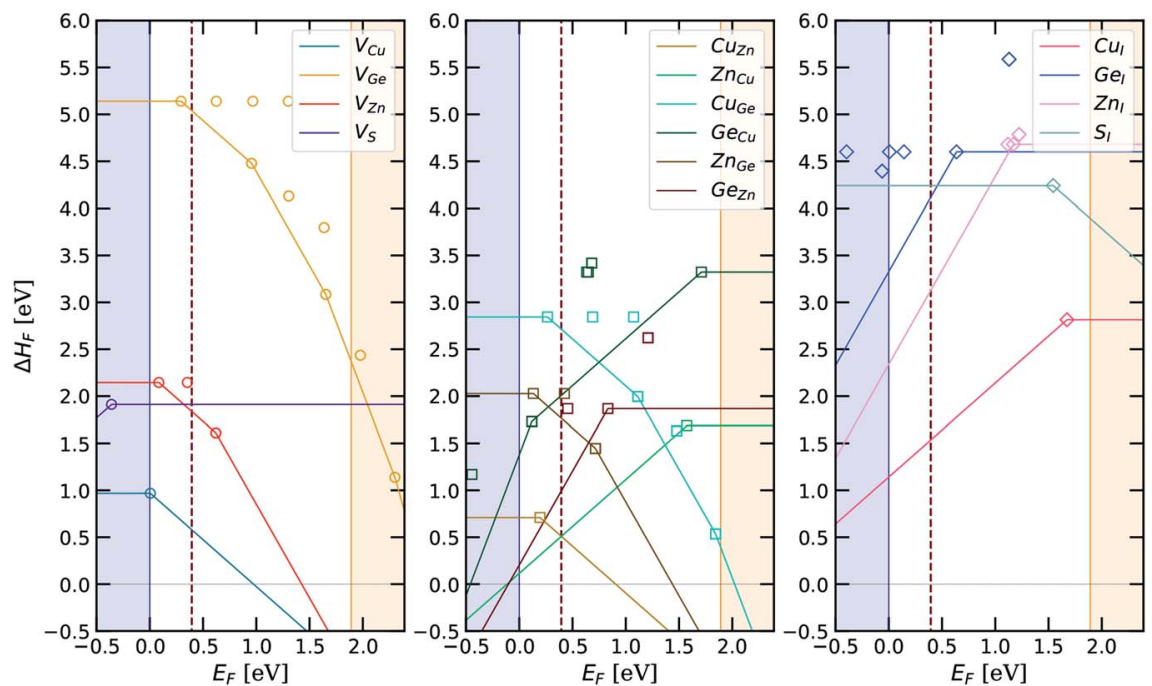

(b) $\mathrm{Cu}_{2} \mathrm{ZnGeS} 4$

Fig. 3 Formation energies of point defects in (a) $\mathrm{Cu}_{2} \mathrm{ZnSnS}_{4}$ and (b) $\mathrm{Cu}_{2} \mathrm{ZnGeS}_{4}$ for three different kinds of defects using a particular marker: vacancy $V_{X}$ (circle), substitution $X_{Y}$ (square) and interstitial $X_{I}$ (diamond) calculated at the chemical potential point $E$ corresponding to $\mu_{C u}=-0.55$ $\mathrm{eV}, \mu_{\mathrm{Zn}}=-1.56 \mathrm{eV}, \mu_{\mathrm{Sn}}=-0.56 \mathrm{eV}$ and $\mu_{\mathrm{Ge}}=-0.79 \mathrm{eV}$ for the Sn-kesterite and $\mu_{\mathrm{Cu}}=-0.55 \mathrm{eV}, \mu_{\mathrm{Zn}}=-1.506 \mathrm{eV}$ and $\mu_{\mathrm{Ge}}=-0.675 \mathrm{eV}$ for the Ge-compound. The Fermi level under thermodynamic equilibrium conditions is represented by the maroon dashed lines (see the ESI $\dagger$ ). Extrinsic defects corresponding to Ge doping in the Sn-based kesterite matrix are represented using a star marker. A specific color is attributed to each defect and each marker corresponds to a possible transition level between two different charge states of the same defect.

explained by the lattice distortion cost induced by the interstitial incorporation. As can be observed, the lowest formations energies (below $1.5 \mathrm{eV}$ ) are reported for the copper vacancy $\mathrm{V}_{\mathrm{Cu}}$, and for $\mathrm{Cu}_{\mathrm{Zn}}, \mathrm{Sn}_{\mathrm{Zn}}$ and $\mathrm{Zn}_{\mathrm{Cu}}$ substitutional defects. In Fig. $3 \mathrm{~b}$ we report the same trend concerning the behaviour of the intrinsic point defect formation energies in the Ge-based compound, highlighting the similarity of intrinsic defects in both kesterites.

In addition, as presented in Fig. 3 and described in the ESI, $\uparrow$ the Fermi level under thermodynamic equilibrium conditions can be extracted. As shown, in both kesterites, under equilibrium conditions the Fermi energy is pinned mainly by the charged defects $\mathrm{V}_{\mathrm{Cu}}$ and $\mathrm{Cu}_{\mathrm{Zn}}$, both being in a charge state of -1 thus providing holes for the electrical conductivity while the substitutional defect $\mathrm{Zn}_{\mathrm{Cu}}$ is in a charge state of +1 , supplying electrons. Under equilibrium, the extracted Fermi energy level value is located at $0.468 \mathrm{eV}$ and $0.409 \mathrm{eV}$ above the VBM, respectively for $\mathrm{Cu}_{2} \mathrm{ZnSnS}_{4}$ and $\mathrm{Cu}_{2} \mathrm{ZnGeS}_{4}$. Through this observation, we consequently highlight the p-type conductivity of $\mathrm{Cu}_{2} \mathrm{ZnSnS}_{4}$, a well-established experimental fact, also confirmed theoretically by Chen et al. ${ }^{6}$ More interestingly, we highlight the same behaviour for the Ge-based kesterite. To complete these observations, we also report the substitutional defect $\mathrm{Sn}_{\mathrm{Zn}}$ (resp. $\mathrm{Ge}_{\mathrm{Zn}}$ ) which would be in a charge state +2 . 
However as will be described later on, $\mathrm{Sn}_{\mathrm{Zn}}$ (resp. $\mathrm{Ge}_{\mathrm{Zn}}$ ) would more likely behave as a recombination centre.

Then, we present Ge-doping of extrinsic point defects in the Sn-kesterite matrix such as $\mathrm{Ge}_{\mathrm{Cu}}, \mathrm{Ge}_{\mathrm{Zn}}$, and $\mathrm{Ge}_{\mathrm{Sn}}$ substitutional defects and the $\mathrm{Ge}_{\mathrm{I}}$ interstitial defect. As shown in Fig. 3a, both $\mathrm{Ge}_{\mathrm{Cu}}$ and $\mathrm{Ge}_{\mathrm{I}}$ have high formation energies with values above 2 $\mathrm{eV}$. This result highlights the low probability for the Ge element to be incorporated in the Sn-kesterite via $\mathrm{Cu}$ substitution or as an interstitial defect. In contrast, the substitutional defect $\mathrm{Ge}_{\mathrm{Zn}}$ presents a formation energy below $2 \mathrm{eV}$, whereas $\mathrm{Ge}_{\mathrm{Sn}}$ has a formation energy below $0.5 \mathrm{eV}$, meaning that this defect could form quite spontaneously in the presence of Ge. It is also interesting to note that in Fig. 3a, if one compares $\mathrm{Ge}_{\mathrm{Cu}}$ and $\mathrm{Sn}_{\mathrm{Cu}}$ (resp. $\mathrm{Ge}_{\mathrm{Zn}}$ and $\mathrm{Sn}_{\mathrm{Zn}}$ ), both extrinsic doping substitutional defects present higher formation energies than the intrinsic ones. It is also worth noting that the Ge chemical potential $\mu_{\mathrm{Ge}}$ used to compute the Ge-doping defects corresponds to the richest composition value leading consequently to the lowest formation energies. As a result, for a Ge chemical potential value lower than $\mu_{\mathrm{Ge}}=-0.79 \mathrm{eV}$ (poorer composition), the Ge-related defect formation energies will increase (see the ESI $\dagger$ ).

However, depending on the growth conditions of the materials, the defect formation energies will vary. Indeed, for a lower concentration of copper during the materials growth, $\mathrm{Cu}$ vacancies will form with a greater ease and will consequently be present in a higher concentration. This behaviour is captured by the lowering of the formation energy following the change of the $\mathrm{Cu}$ chemical potential value in eqn (1). To visualise these trends, as shown in Fig. 4, the defect formation energies were represented for different hypothetical growth conditions. To do so, we represent the evolution of the formation energies following a specific path in the phase diagrams presented in Fig. 2 and for a Fermi energy value under thermodynamic equilibrium conditions (see the ESI $\dagger$ ). As presented in the phase diagrams of the Sn- and the Ge-kesterite, the chemical potential path studied here is labelled as A-I. The chemical potential coordinates A-D correspond to $\mathrm{Cu}$-rich conditions, $\mathrm{E}-\mathrm{H}$ to $\mathrm{Cu}-$ moderate conditions and finally the I label corresponds to a $\mathrm{Cu}$ poor condition. This path was selected to study the behaviour of the formation energies at the edges of the kesterite pure phases (as represented via the green shading in Fig. 2) and following a Cu concentration from high values to lower ones. The relevant message here is consequently focused on the trends of the defect formation energies for different growth conditions and not specifically on the selected path.

In Fig. 4, we identify $\mathrm{Cu}_{\mathrm{Zn}}{ }^{1-}, \mathrm{Zn}_{\mathrm{Cu}}{ }^{1+}, \mathrm{Sn}_{\mathrm{Zn}}{ }^{2+}$ (resp. $\mathrm{Ge}_{\mathrm{Zn}}{ }^{2+}$ ) and $\mathrm{V}_{\mathrm{Cu}}{ }^{1-}$ as the intrinsic point defects showing the lowest formation energies over the different chemical potential combinations. Following these results, the usually observed $\mathrm{Cu}$ / $\mathrm{Zn}$ disorder in Sn-kesterite can also be expected in the Ge-kesterite as the two substitutional defects $\mathrm{Cu}_{\mathrm{Zn}}{ }^{1-}$ and $\mathrm{Zn}_{\mathrm{Cu}}{ }^{1+}$ present formation energies below $1 \mathrm{eV}$ for any chemical potential combination. This result is in good agreement with those described by Chen et al. where $\mathrm{Cu}_{\mathrm{Zn}}{ }^{1-}$ is the dominant point defect. ${ }^{6}$ In addition, we report a lower formation energy concerning the $\mathrm{Zn}_{\mathrm{Cu}}{ }^{1+}$ antisite with a formation energy between

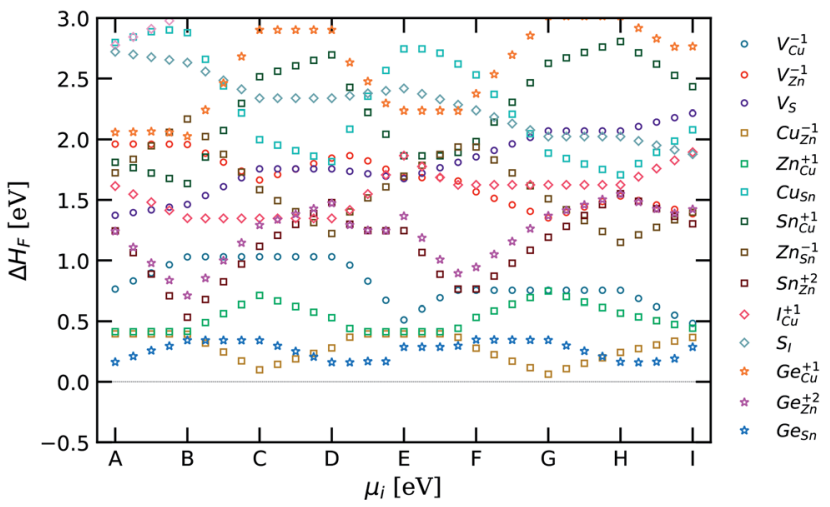

(a) $\mathrm{Cu}_{2} \mathrm{ZnSnS}_{4}$

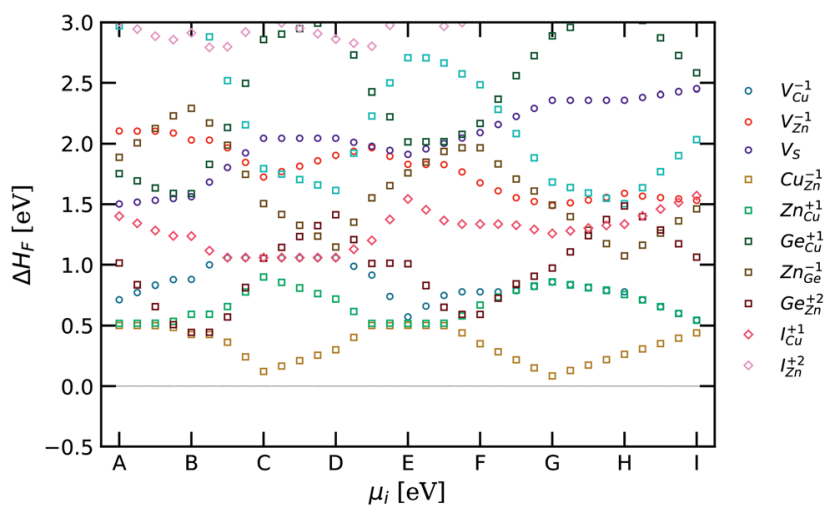

(b) $\mathrm{Cu}_{2} \mathrm{ZnGeS}_{4}$

Fig. 4 Evolution of the point defect formation energies in (a) $\mathrm{Cu}_{2}-$ $\mathrm{ZnSnS}_{4}$ and (b) $\mathrm{Cu}_{2} \mathrm{ZnGeS}_{4}$ for different hypothetical growth conditions. The calculations were performed at various chemical potential combinations corresponding to the label points presented in Fig. 2 (see the ESI $\dagger$ for specific values) and for the Fermi energy levels under thermodynamic equilibrium conditions located at $0.468 \mathrm{eV}$ and 0.409 $\mathrm{eV}$ above the VBM respectively for $\mathrm{Cu}_{2} \mathrm{ZnSnS}_{4}$ and $\mathrm{Cu}_{2} \mathrm{ZnGeS}_{4}$. Each defect is represented using a specific color and using the following markers: circles for intrinsic vacancies, squares for intrinsic substitutional defects, diamonds for intrinsic interstitials and stars for $\mathrm{Ge}$ extrinsic doping defects in the Sn-based kesterite.

$0.03 \mathrm{eV}$ and $0.63 \mathrm{eV}$ while this previous work predicted a formation energy between 0.6 and $0.9 \mathrm{eV}$. As a result, our calculations predict a substitutional defect $\mathrm{Zn}_{\mathrm{Cu}}{ }^{1+}$ concentration within the same range as the $\mathrm{Cu}_{\mathrm{Zn}}{ }^{1-}$ defect. This observation is in good agreement with the work of Du et al. reporting a similar $\mathrm{Zn}_{\mathrm{Cu}}{ }^{1+}$ behaviour. ${ }^{33}$ The facilitated formation of these defects could be explained by the similar atomic radii of $\mathrm{Zn}\left(r_{\mathrm{Zn}}\right.$ $=1.35 \AA)$ and $\mathrm{Cu}\left(r_{\mathrm{Zn}}=1.35 \AA\right)$ while their electronic configurations differ only by one electron. ${ }^{57}$ Several studies report that the related $\mathrm{Cu} / \mathrm{Zn}$ disorder defects are responsible for potential fluctuations detrimental to solar cell performances ${ }^{58,59}$ which could however be suppressed via $\mathrm{Ag}$ incorporation. ${ }^{38}$

As shown in Fig. 4a, focusing on the Ge extrinsic doping defects, the substitutional defect $\mathrm{Ge}_{\mathrm{Sn}}$ presents formation energy values below $0.5 \mathrm{eV}$ for every chemical potential combination. The smaller atomic radius of $\mathrm{Ge}\left(r_{\mathrm{Ge}}=1.25 \AA\right.$ in 
comparison to the Sn element $\left(r_{\mathrm{Sn}}=1.45 \AA\right)$ and their similar electronic behaviours could explain the high occurrence of this defect. Then, a general trend observed, moving from the Snbased kesterite to the Ge-doped kesterite, is the increase of the formation energy from the intrinsic defect $\mathrm{Sn}_{\mathrm{Zn}}{ }^{2+}$ with $\Delta H_{\mathrm{F}}=$ $1.24 \mathrm{eV}$ to $\mathrm{Ge}_{\mathrm{Zn}}{ }^{2+}$ with $\Delta H_{\mathrm{F}}=1.34 \mathrm{eV}$ (one should also note that for some chemical potentials, those quantities can be equal). As reported in Fig. 3, an increase of the formation energy is also observed as we move from the neutral defect $\mathrm{Sn}_{\mathrm{Zn}}$ in the Snbased kesterite to the Ge-doped and then Ge-alloyed kesterite defect $\mathrm{Ge}_{\mathrm{Zn}}$ with values of 1.45, 1.79 and $1.85 \mathrm{eV}$ respectively. This implies that if Ge is available, the ease of formation of the Zn substitutional defect would be further reduced, first in the Sn-kesterite following Ge doping and then in the Ge-kesterite upon complete substitution of Sn. In their study, Wexler et al. reported an increase of the $2 \mathrm{Cu}_{\mathrm{Zn}}+\mathrm{X}_{\mathrm{Zn}}$ defect cluster formation energy following the complete Sn substitution. ${ }^{28}$ This observation was attributed to the multivalence of Sn which can reduce from a $4+$ to a $2+$ oxidation state. This change of oxidation allows $\mathrm{Sn}$ to exist in a +2 state on the $\mathrm{Zn}^{2+}$ site. In contrast, $\mathrm{Ge}$ only exists in a 4+ state explaining the formation energy increase between the $\mathrm{Ge}_{\mathrm{Zn}}$ and the $\mathrm{Sn}_{\mathrm{Zn}}$ substitutional defects. ${ }^{15,16,28}$

Here, we have reported $\mathrm{Cu}_{\mathrm{Zn}}{ }^{1-}, \mathrm{Zn}_{\mathrm{Cu}}{ }^{1+} ., \mathrm{Sn}_{\mathrm{Zn}}{ }^{2+}\left(\right.$ resp. $\left.\mathrm{Ge}_{\mathrm{Zn}}{ }^{2+}\right)$ and $\mathrm{V}_{\mathrm{Cu}}{ }^{1-}$ as the most abundant point defects in $\mathrm{Cu}_{2} \mathrm{ZnSnS}_{4}$ (resp. $\mathrm{Cu}_{2} \mathrm{ZnGeS}_{4}$ ). In the next section, we focus on their physical behaviour: acceptors, donors or recombination centres.

\subsection{Defect identification: ionisation levels}

In Fig. 5, we show the ionisation levels for each defect according to eqn (2). It is important to note that these levels within the kesterite band gap are, in contrast to the formation energies, independent of the chemical potential values. The relevant information here is (i) the position of the ionisation level in the material band gap, which determines the behaviour of the defect, and (ii) as a guide for the eye, the "formation energy value" of the ionisation energy level $\beta$ for a Fermi level located at the transition level within the kesterite band gap (see also markers in Fig. 3).

For both $\mathrm{Cu}_{2} \mathrm{ZnSnS}_{4}$ and $\mathrm{Cu}_{2} \mathrm{ZnGeS}_{4}$, as shown Fig. 5, we highlight the $(0 /-1)$ transition levels of $\mathrm{Cu}_{\mathrm{Zn}}$ and $\mathrm{V}_{\mathrm{Cu}}$ located at a few $k_{\mathrm{B}} T$ over the VBM. From the calculation of the Fermi energy under thermodynamic equilibrium conditions, these transition levels are ionised and consequently, these defects act as acceptors both providing holes to the kesterite conductivity. Combined with their low formation energies, these levels are particularly suited to account for the p-type intrinsic conductivity of these compounds. Then, as donor defects, we report the $(+1 / 0)$ transition level of the substitutional defect $\mathrm{Zn}_{\mathrm{Cu}}$ located close to the conduction band minimum (CBM). With its previously reported low formation energy, this defect is the most abundant donor in both kesterites. We can also note that in the case of the Ge-compound, in opposition to the Sn-kesterite, the $(+1 / 0)$ transition level of $\mathrm{Zn}_{\mathrm{Cu}}$ is below the CBM. This is a direct consequence of the band gap increase associated with the Ge alloying. The pinning of the Fermi level under thermodynamic

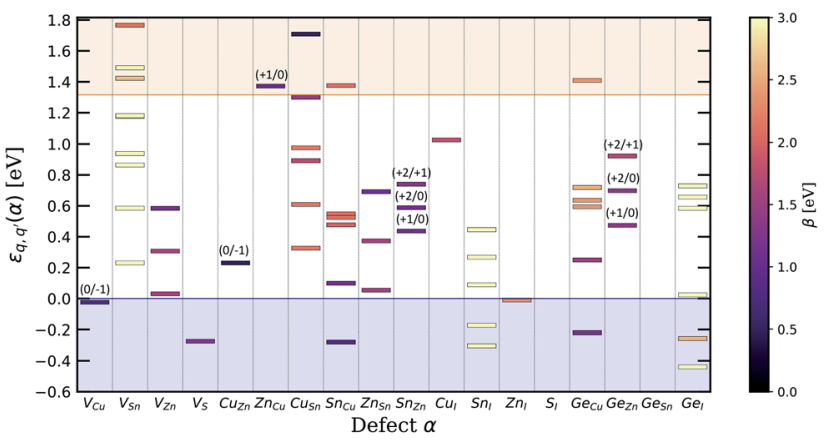

(a) $\mathrm{Cu}_{2} \mathrm{ZnSnS}_{4}$

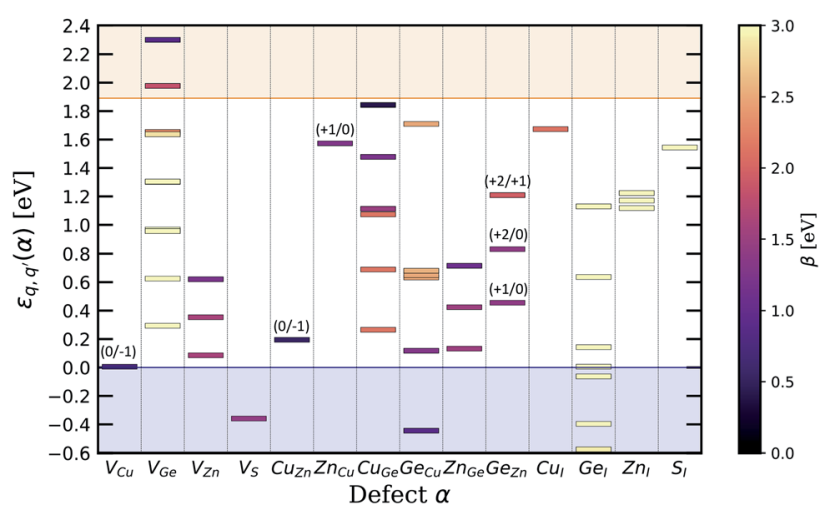

(b) $\mathrm{Cu}_{2} \mathrm{ZnGeS}_{4}$

Fig. 5 lonisation energy levels of intrinsic point defects in (a) $\mathrm{Cu}_{2}-$ $\mathrm{ZnSnS}_{4}$ and (b) $\mathrm{Cu}_{2} \mathrm{ZnGeS}_{4}$ calculated for a chemical potential combination corresponding to point $\mathrm{E}$ in Fig. $2 \mathrm{~b}$ and e. Their locations within the kesterite band gaps are reported here on the $y$-axis while the $\beta$ value corresponds to the formation energy value at which the transition occurs in Fig. 3. This $\beta$ value is provided as a guide for the eye to highlight the most dominant transition levels.

equilibrium conditions is a result of these two observations, which explain the p-type conductivity of kesterite materials generally reported in the literature. ${ }^{6} \mathrm{We}$ also note that the $\mathrm{V}_{\mathrm{Zn}}$ defect provides energy levels close to the VBM; however, assuming a p-type material, the Fermi level would be close to the valence band leading to an unionised defect with a high formation energy as presented in Fig. 3a. Concerning the substitutional defect $\mathrm{Sn}_{\mathrm{Cu}}$ (resp. $\mathrm{Ge}_{\mathrm{Cu}}$ ), it presents ionisation states not suited for intrinsic doping (i.e. "donor states" close to the valence band). A similar behaviour is observed for the substitutional defect $\mathrm{Cu}_{\mathrm{Sn}}$. As a consequence, these defects should not contribute significantly to conductivity in either of the kesterites.

One can identify $\mathrm{Sn}_{\mathrm{Zn}}\left(\right.$ resp. $\left.\mathrm{Ge}_{\mathrm{Zn}}\right), \mathrm{Zn}_{\mathrm{Sn}}\left(\right.$ resp. $\left.\mathrm{Zn}_{\mathrm{Ge}}\right)$ and to a lesser extent $\mathrm{Cu}_{\mathrm{Sn}}\left(\right.$ resp. $\left.\mathrm{Cu}_{\mathrm{Ge}}\right)$ and $\mathrm{Sn}_{\mathrm{Cu}}\left(\right.$ resp. $\mathrm{Ge}_{\mathrm{Cu}}$ ) as deep defects. Indeed, all these substitutional defects offer transition levels located close to the middle of the kesterite band gap. In addition, as presented in Fig. 4, the substitution of $\mathrm{Zn}$ by $\mathrm{Sn}$ (resp. Ge) is the only defect presenting also a low formation energy. The latter has several transition levels within the kesterite band gap corresponding to the various transitions between its various charge states: $(+2 / 0)$ at $0.584 \mathrm{eV}$ (resp. 0.736 $\mathrm{eV}),(+2 /+1)$ at $0.735 \mathrm{eV}($ resp. $0.585 \mathrm{eV})$ and $(+1 / 0)$ at $0.43 \mathrm{eV}$ 
(resp. $0.890 \mathrm{eV}$ ) above the VBM (below the CBM). These values are comparable to those reported by Li et al. with $\mathrm{Sn}_{\mathrm{Zn}}(+1 / 0)$ at $0.86 \mathrm{eV}$ and $(+2 / 0)$ at $0.67 \mathrm{eV}$ below the CBM..$^{43}$ Moreover, as a result of the p-type conductivity, the $\mathrm{Sn}_{\mathrm{Zn}}\left(\right.$ resp. $\left.\mathrm{Ge}_{\mathrm{Zn}}\right)$ defect should be ionised into a charge state +2 , with the most probable transition energy level being the two-electron transition $(+2 / 0)$ (see Fig. 3). Using deep-level transient spectroscopy, Deng et al. reported a defect activation energy of $0.581 \mathrm{eV}$ for the Sn-based kesterite, and identified this defect as a recombination centre. ${ }^{36}$ As shown in Fig. 5a and in agreement with this experimental observation, this defect could be reported as the substitutional defect $\mathrm{Sn}_{\mathrm{Zn}}$ with its two-electron transition level $(+2 / 0)$ at 0.584 $\mathrm{eV}$ above the VBM. Furthermore, the same authors reported a decrease of the transition level position to $0.542 \mathrm{eV}$ following Ge incorporation, while in this work, upon Ge incorporation we report the spreading of the ionisation levels located between $0.42 \mathrm{eV}$ and $0.73 \mathrm{eV}$ for $\mathrm{Sn}_{\mathrm{Zn}}$, between $0.46 \mathrm{eV}$ and $0.91 \mathrm{eV}$ for $\mathrm{Ge}_{\mathrm{Zn}}$ (in Sn-based kesterite) and between $0.44 \mathrm{eV}$ and $1.9 \mathrm{eV}$ for $\mathrm{Ge}_{\mathrm{Zn}}$ (in Ge-based kesterite). It should also be noted that in all three situations, the transition level $(+2 / 0)$ is the closest to the middle of the gap.

Beyond formation energies, other physical parameters can also act as indicators for the evaluation of the impact of point defects on solar cell properties. In the following section, we consider recombination centre capture cross sections, which can be related to the kesterite lattice distortion upon introduction of defects.

\subsection{Atomic distortions}

In Fig. 6, we present the evolution of the interatomic distances between the defect position and the surrounding $\mathrm{S}$ atoms with respect to the charge states $q$ of the defect, both in the Sn-based and the Ge-based compounds. As reported by Li et al. ${ }^{43}$ an empirical approach to evaluate the carrier capture cross section of defects consists in studying the local structural relaxation undergone by the lattice when the defect captures/emits elec$\operatorname{tron}(\mathrm{s}) .{ }^{43}$ They suggested that strong bonds and large structural relaxations imply large defect capture cross sections. Such a qualitative approach can be more firmly grounded using a quantitative study of the defect carrier capture cross section via the computation of the phonon-electron Hamiltonian. ${ }^{\mathbf{1 6 , 4 3 , 6 0}}$ As expressed in eqn (3), the emission/capture rate of a defect does not depend solely on the capture cross section. Indeed, the electron emission rate $e_{\mathrm{n}}$ (resp. $e_{\mathrm{p}}$ for holes) is related to its ionisation level in the kesterite band gap with respect to the CBM: $E_{\mathrm{C}}-E_{\mathrm{t}}$ (resp. to the VBM: $E_{\mathrm{t}}-E_{\mathrm{V}}$ for holes) and to the capture cross section of the defect $\sigma_{\mathrm{n}}$ (resp. $\sigma_{\mathrm{p}}$ ) following this relation:

$$
e_{\mathrm{n}}=\sigma_{\mathrm{n}}\left\langle v_{\mathrm{n}}\right\rangle N_{\mathrm{C}} \exp \left(-\frac{E_{\mathrm{C}}-E_{\mathrm{t}}}{k_{\mathrm{B}} T}\right),
$$

where $\left\langle v_{\mathrm{n}}\right\rangle$ (resp. $\left\langle v_{\mathrm{p}}\right\rangle$ ) is the average thermal velocity of the electron (resp. hole) and $N_{\mathrm{C}}$ (resp. $N_{\mathrm{V}}$ ) the effective density of states in the conduction band (resp. valence band).

A first general trend that can be observed for each defect is that as the charge state of the defect gets closer to the electronic
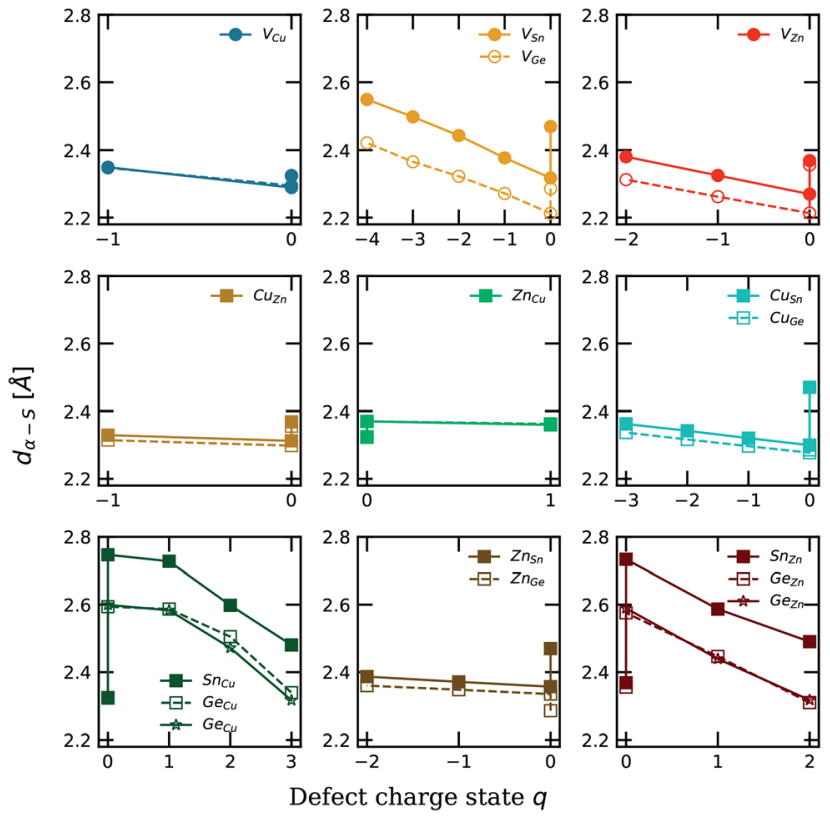

Fig. 6 Representation of the interatomic distance between the defect position and the surrounding $S$ atoms with respect to the defect charge state $q$. For a charge state $q=0$, the evolution corresponds to the distortion undergone by the lattice following the defect incorporation while the evolution along the $x$-axis corresponds to the lattice distortion as a result of the capture/emission of electrons by the defect. The results corresponding to the $\mathrm{Cu}_{2} \mathrm{ZnSnS}_{4}\left(\right.$ resp. $\mathrm{Cu}_{2} \mathrm{ZnGeS}_{4}$ ) compound are reported in plain (resp. dashed) lines. Ge-doping distortions in Sn-kesterite are reported in plain lines using a star symbol.

configuration of the pristine system, the lattice distortion tends to get closer to the reference distance. For example, in the case of a substitutional defect, if both elements have similar atomic radii and if their electronic configurations are close, one could expect the lattice to undergo a small distortion upon defect incorporation.

A similar behaviour is observed in both $\mathrm{Cu}_{2} \mathrm{ZnSnS}_{4}$ and $\mathrm{Cu}_{2} \mathrm{ZnGeS}_{4}$ concerning defects providing transition levels close to the band gap edges and showing low formation energies $\left(\mathrm{Cu}_{\mathrm{Zn}}, \mathrm{Zn}_{\mathrm{Cu}}\right.$ and $\left.\mathrm{V}_{\mathrm{Cu}}\right)$. The introduction of the copper vacancy leads only to a small distortion of the lattice as the interatomic distance variation is less than $0.1 \AA$. In addition, assuming the capture of an electron by such a defect, the value of the interatomic distance remains quite constant in comparison to the one in the pristine lattice. This result supports the readiness of these defects to provide charge carriers in kesterite materials. We report the same behaviour for the substitutional defects $\mathrm{Cu}_{\mathrm{Zn}}$ and $\mathrm{Zn}_{\mathrm{Cu}}$. The results also support that the $\mathrm{Cu} / \mathrm{Zn}$ disorder commonly observed in Sn-based kesterites should be present in the Ge-based compound as well. In addition, for the $\mathrm{Ge}_{\mathrm{Sn}}$ substitutional defect in the Ge-based kesterite, we report a reduction of the $\mathrm{X}-\mathrm{S}(\mathrm{X}=\mathrm{Sn}, \mathrm{Ge})$ interatomic distance from $2.46 \AA$ in the Sn-based compound to $2.30 \AA$ in the Ge-based material. This can be interpreted as a direct consequence of the smaller atomic radius of $\mathrm{Ge}\left(r_{\mathrm{Ge}}=1.25 \AA\right.$ compared to the $\mathrm{Sn}$ element: $r_{\mathrm{Sn}}=1.45 \AA$ (ref. $\left.{ }^{57}\right)$ ). 
Moreover, we observe the largest lattice distortions for both substitutional defects $\mathrm{Sn}_{\mathrm{Cu}}$ (resp. $\mathrm{Ge}_{\mathrm{Cu}}$ ) and $\mathrm{Sn}_{\mathrm{Zn}}$ (resp. $\mathrm{Ge}_{\mathrm{Zn}}$ ) which were identified as possible recombination centres in the previous section. In good agreement with our calculations, $\mathrm{Li}$ et al. reported similar values concerning the $\mathrm{Sn}-\mathrm{S}$ distances for various charged states of the $\mathrm{Sn}_{\mathrm{Zn}}$ defect, i.e. $2.71 \AA(q=0), 2.57$ $\AA(q=1)$ and $2.43 \AA(q=2)$ while the values obtained in this work are $2.73 \AA$, $2.59 \AA$ and $2.49 \AA$ respectively for $q=0,1$ and 2 . In addition, the distortion inside the Sn-kesterite is stronger than that found in the Ge-based kesterite. Following the empirical rule proposed by Li et al. in ref. ${ }^{43}$, this would lead to a smaller carrier capture cross section in the Ge-compound, partially explaining the smaller $V_{\text {OC }}$ deficit reported in the Gebased kesterite with respect to the Sn-based material.

To summarise, following the Ge incorporation in the Snkesterite, from Ge-doping to pure Ge-alloyed materials, we report an increase of the neutral $\mathrm{X}_{\mathrm{Zn}}(\mathrm{X}=\mathrm{Sn}, \mathrm{Ge})$ substitutional defect formation energies which consequently results in a decrease of the defect concentrations. Secondly, along with this observation, the ionisation levels associated with the $\mathrm{X}_{\mathrm{Zn}}(\mathrm{X}$ $=\mathrm{Sn}, \mathrm{Ge}$ ) defect tend to spread within the material band gap as we move from Ge doping to Ge alloying with the $(+2 / 0)$ transition level still located in the middle of the gap. Finally, from the lattice distortion investigation, we report a reduction of the $\mathrm{X}_{\mathrm{Zn}^{-}}$ $\mathrm{S}(\mathrm{X}=\mathrm{Sn}, \mathrm{Ge})$ interatomic distance, which results in a decrease of the associated carrier capture cross section. In addition to these observations, we previously reported an increase of the $V_{\text {OC }}$ value as well as a decrease of the $J_{\mathrm{SC}}$ value as a result of the increase of the material band gap from $1.32 \mathrm{eV}\left(\mathrm{Cu}_{2} \mathrm{ZnSnS}_{4}\right)$ to $1.89 \mathrm{eV}\left(\mathrm{Cu}_{2} \mathrm{ZnGeS}_{4}\right){ }^{40}$ Combining the results from both investigations, we thus conclude that the improvement of the solar cell efficiency associated with the Ge doping of Sn-based kesterites is ascribed to the improvement of the $V_{\mathrm{OC}}$ value while maintaining the $J_{\mathrm{SC}}$ value of the Sn-based kesterite cell. In comparison to the Sn counterpart, this enhancement is the result of both the increase of the $\mathrm{Ge}_{\mathrm{Zn}}$ substitutional defect formation energy and the reduction of its associated carrier capture cross section. As reported in several studies, both of these observations can be related to the ability of $\mathrm{Sn}$ to reduce its oxidation state from $4+$ to $2+$ known as the "lone-pair" effect while, in contrast, Ge possess only one oxidation state which is $4+.{ }^{15,16,42}$

\section{Conclusion}

This work was devoted to first-principles investigations of Gerelated defects in kesterite. First, we highlight the slightly wider pure phase range of chemical potential values of the Ge-based kesterite compared to the Sn-based kesterite. In both cases, the pure phase remains limited in terms of possible chemical potential ranges due to the numerous secondary phases, which are a direct consequence of all four elements present in these kesterites. Near the stable phase region, the secondary phases are $\mathrm{ZnS}, \mathrm{CuS}$ and $\mathrm{Cu}_{2} \mathrm{SnS}_{3}$ (resp. $\mathrm{Cu}_{2} \mathrm{GeS}_{3}$ ) in the Sn-kesterite (resp. Ge-kesterite). We found a similar physical behaviour of the intrinsic point defects for the Sn-kesterite and the Ge-kesterite. In both compounds, we identified $\mathrm{V}_{\mathrm{Cu}}{ }^{1-}, \mathrm{Cu}_{\mathrm{Zn}}{ }^{1-}$ and
$\mathrm{Zn}_{\mathrm{Cu}}{ }^{1+}$ as low formation energy defects acting as acceptors and as donor defects for the latter. By calculating the Fermi level under equilibrium conditions, we confirmed the p-type conductivity reported in the literature for both the Sn-based and the Ge-based compounds. We also shed light on the commonly observed $\mathrm{Cu} / \mathrm{Zn}$ disorder encountered in kesterite compounds. In addition, via the study of Ge doping in the Sn-kesterite compound, we identified the $\mathrm{Ge}_{\mathrm{Sn}}$ neutral defect as a quite spontaneous defect, an indication that the Ge doping within the kesterite matrix occurs via a Sn substitution. Finally, we identified $\mathrm{Sn}_{\mathrm{Zn}}$ and $\mathrm{Ge}_{\mathrm{Zn}}$ as recombination centres. We reported an increase of the substitutional defect formation energy following Ge-doping and alloying which would result in a decrease of the defect concentration. Moreover, it appeared that the lattice distortion induced by the formation of these defects is reduced for the $\mathrm{Ge}_{\mathrm{Zn}}$ substitutional defect in comparison to its $\mathrm{Sn}$ counterpart. This result hints at a reduced carrier capture cross section and consequently a less detrimental defect behaviour ascribed to $\mathrm{Ge}_{\mathrm{Zn}}$ with respect to $\mathrm{Sn}_{\mathrm{Zn}}$. As a consequence, we point out the reduction of the non-radiative recombination rate induced by both the decrease of the concentration and the reduction of the carrier capture cross section of the detrimental defect $\mathrm{X}_{\mathrm{Zn}}(\mathrm{X}=\mathrm{Sn}, \mathrm{Ge})$ as one of the sources of the $V_{\mathrm{OC}}$ improvement reported in the literature upon Ge incorporation.

The objective of this work was to strengthen the understanding of the effects of Ge doping and Ge alloying in kesterite materials for photovoltaic applications. We believe that our results clarified the fundamental mechanisms that operate at the atomic scale via the formation of a wide range of point defects and linked them to photovoltaic property enhancement of Ge-based kesterite reported in previous studies.

\section{Author contributions}

All authors contributed to the elaboration of the study and the main conceptual ideas. The data production and analysis, the figure elaboration and the interpretations as well as the writing, editing and review of the manuscript were realised by TR. JY and NDN contributed to the result interpretations, the final discussions and the review of the manuscripts. GB and BV contributed to the result discussion and the final manuscript review.

\section{Conflicts of interest}

There are no conflicts to declare.

\section{Acknowledgements}

Computational resources have been provided by the Consortium des Équipements de Calcul Intensif (CÉCI), funded by the Fonds de la Recherche Scientifique de Belgique (F. R. S.-FNRS) under Grant No. 2.5020.11 and by the Walloon Region. J. Y. R. thanks the F. R. S.-FNRS for the ABIGLO grant J.0154.21. N. D. N. acknowledges the financial support by the F. R. S.-FNRS of Belgium (project J.0124.19). We would also like to acknowledge Robert B. Wexler and the research group of Prof. Emily Carter 
for the fruitful discussions shared concerning Ge-related defects in kesterite materials.

\section{Notes and references}

1 M. Green, E. Dunlop, J. Hohl-Ebinger, M. Yoshita, N. Kopidakis and X. Hao, Prog. Photovoltaics Res. Appl., 2021, 29, 3-15.

2 M. Nakamura, K. Yamaguchi, Y. Kimoto, Y. Yasaki, T. Kato and H. Sugimoto, IEEE J. Photovolt., 2019, 9, 1863-1867.

3 S. Giraldo, Z. Jehl, M. Placidi, V. Izquierdo-Roca, A. PérezRodríguez and E. Saucedo, Adv. Mater., 2019, 31, 1806692.

4 T. Ratz, G. Brammertz, R. Caballero, M. León, S. Canulescu, J. Schou, L. Gütay, D. Pareek, T. Taskesen, D.-H. Kim, J. K. Kang, C. Malerba, A. Redinger, E. Saucedo, B. Shin, H. Tampo, K. Timmo, N. D. Nguyen and B. Vermang, $J$. Phys.: Energy, 2019, 1, 042003-042024.

5 T. Todorov, H. Hillhouse, S. Aazou, Z. Sekkat, O. Vigil-Galán, S. Deshmukh, R. Agrawal, S. Bourdais, M. Valdés, P. Arnou, et al., J. Phys.: Energy, 2020, 2, 012003.

6 S. Chen, A. Walsh, X.-G. Gong and S.-H. Wei, Adv. Mater., 2013, 25, 1522-1539.

7 S. Kim, J.-S. Park and A. Walsh, ACS Energy Lett., 2018, 3, 496500.

8 M. Grossberg, J. Krustok, C. J. Hages, D. M. Bishop, O. Gunawan, R. Scheer, S. M. Lyam, H. Hempel, S. Levcenco and T. Unold, J. Phys.: Energy, 2019, 1, 044002.

9 K. Pal, P. Singh, A. Bhaduri and K. B. Thapa, Sol. Energy Mater. Sol. Cells, 2019, 196, 138-156.

10 M. Dimitrievska, A. Fairbrother, E. Saucedo, A. PérezRodríguez and V. Izquierdo-Roca, Sol. Energy Mater. Sol. Cells, 2016, 149, 304-309.

11 S. Schorr, G. Gurieva, M. Guc, M. Dimitrievska, A. PérezRodríguez, V. Izquierdo-Roca, C. S. Schnohr, J. Kim, W. Jo and J. M. Merino, J. Phys.: Energy, 2019, 2, 012002.

12 C. Platzer-Björkman, N. Barreau, M. Bär, L. Choubrac, L. Grenet, J. Heo, T. Kubart, A. Mittiga, Y. Sánchez, J. Scragg, S. Sinha and M. Valentini, J. Phys.: Energy, 2019, 1, 044005-044022.

13 A. Crovetto and O. Hansen, Sol. Energy Mater. Sol. Cells, 2017, 169, 177-194.

14 T. Gokmen, O. Gunawan, T. K. Todorov and D. B. Mitzi, Appl. Phys. Lett., 2013, 103, 103506.

15 K. Biswas, S. Lany and A. Zunger, Appl. Phys. Lett., 2010, 96, 201902.

16 S. Kim, J.-S. Park, S. N. Hood and A. Walsh, J. Mater. Chem. A, 2019, 7, 2686-2693.

17 Y. Gong, Y. Zhang, Q. Zhu, Y. Zhou, R. Qiu, C. Niu, W. Yan, W. Huang and H. Xin, Energy Environ. Sci., 2021, 14, 23692380.

18 Y. E. Romanyuk, S. G. Haass, S. Giraldo, M. Placidi, D. Tiwari, D. J. Fermin, X. Hao, H. Xin, T. Schnabel, M. Kauk-Kuusik, P. Pistor, S. Lie and L. H. Wong, J. Phys.: Energy, 2019, 1, 044004-044023.

19 A. Crovetto, S. Kim, M. Fischer, N. Stenger, A. Walsh, I. Chorkendorff and P. C. Vesborg, Energy Environ. Sci., 2020, 13, 3489-3503.
20 M. V. Jyothirmai, H. Saini, N. Park and R. Thapa, Sci. Rep., 2019, 9, 1-12.

21 J. Li, D. Wang, X. Li, Y. Zeng and Y. Zhang, Adv. Sci., 2018, 5, 1700744.

22 M. Neuschitzer, M. E. Rodriguez, M. Guc, J. A. Marquez, S. Giraldo, I. Forbes, A. Perez-Rodriguez and E. Saucedo, J. Mater. Chem. A, 2018, 6, 11759-11772.

23 Q. Tian and S. F. Liu, J. Mater. Chem. A, 2020, 8, 24920-24942.

24 Z.-K. Yuan, S. Chen, H. Xiang, X.-G. Gong, A. Walsh, J.-S. Park, I. Repins and S.-H. Wei, Adv. Funct. Mater., 2015, 25, 6733-6743.

25 W.-C. Huang, S.-Y. Wei, C.-H. Cai, W.-H. Ho and C.-H. Lai, J. Mater. Chem. A, 2018, 6, 15170-15181.

26 M. H. Sharif, T. Enkhbat, E. Enkhbayar and J. Kim, ACS Appl. Energy Mater., 2020, 3, 8500-8508.

27 S. Hadke, W. Chen, J. M. R. Tan, M. Guc, V. Izquierdo-Roca, G.-M. Rignanese, G. Hautier and L. H. Wong, J. Mater. Chem. A, 2019, 7, 26927-26933.

28 R. B. Wexler, G. S. Gautam and E. A. Carter, J. Mater. Chem. A, 2021, 9, 9882-9897.

29 S. Khelifi, G. Brammertz, L. Choubrac, M. Batuk, S. Yang, M. Meuris, N. Barreau, J. Hadermann, H. Vrielinck, D. Poelman, et al., Sol. Energy Mater. Sol. Cells, 2021, 219, 110824.

30 B. Vermang, G. Brammertz, M. Meuris, T. Schnabel, E. Ahlswede, L. Choubrac, S. Harel, C. Cardinaud, L. Arzel, N. Barreau, et al., Sustainable Energy Fuels, 2019, 3, 22462259.

31 L. Choubrac, M. Bär, X. Kozina, R. Felix, R. G. Wilks, G. Brammertz, S. Levcenko, L. Arzel, N. Barreau, S. Harel, et al., ACS Appl. Energy Mater., 2020, 3, 5830-5839.

32 S. Giraldo, M. Neuschitzer, T. Thersleff, S. López-Marino, Y. Sánchez, H. Xie, M. Colina, M. Placidi, P. Pistor, V. Izquierdo-Roca, et al., Adv. Energy Mater., 2015, 5, 1501070.

33 Y. Du, S. Wang, Q. Tian, Y. Zhao, X. Chang, H. Xiao, Y. Deng, S. Chen, S. Wu and S. Liu, Adv. Funct. Mater., 2021, 31, 2010325.

34 S. Kim, K. M. Kim, H. Tampo, H. Shibata and S. Niki, Appl. Phys. Express, 2016, 9, 102301.

35 S. Giraldo, E. Saucedo, M. Neuschitzer, F. Oliva, M. Placidi, X. Alcobé, V. Izquierdo-Roca, S. Kim, H. Tampo, H. Shibata, et al., Energy Environ. Sci., 2018, 11, 582-593.

36 Y. Deng, Z. Zhou, X. Zhang, L. Cao, W. Zhou, D. Kou, Y. Qi, S. Yuan, Z. Zheng and S. Wu, J. Energy Chem., 2021, 61, 1-7. 37 J. Fu, D. Kou, W. Zhou, Z. Zhou, S. Yuan, Y. Qi and S. Wu, J. Mater. Chem. A, 2020, 8, 22292-22301.

38 M. He, J. Huang, J. Li, J. S. Jang, U. P. Suryawanshi, C. Yan, K. Sun, J. Cong, Y. Zhang, H. Kampwerth, et al., Adv. Funct. Mater., 2021, 2104528.

39 W. Wang, M. T. Winkler, O. Gunawan, T. Gokmen, T. K. Todorov, Y. Zhu and D. B. Mitzi, Adv. Energy Mater., 2014, 4, 1301465.

40 T. Ratz, J.-Y. Raty, G. Brammertz, B. Vermang and N. D. Nguyen, J. Phys.: Energy, 2021, 3, 035005.

41 H. Nishihara, T. Maeda, A. Shigemi and T. Wada, Jpn. J. Appl. Phys., 2017, 56, 04CS08. 
42 R. B. Wexler, G. S. Gautam and E. A. Carter, Phys. Rev. B, 2020, 102, 054101.

43 J. Li, Z.-K. Yuan, S. Chen, X.-G. Gong and S.-H. Wei, Chem. Mater., 2019, 31, 826-833.

44 C. Freysoldt, B. Grabowski, T. Hickel, J. Neugebauer, G. Kresse, A. Janotti and C. G. Van de Walle, Rev. Mod. Phys., 2014, 86, 253.

45 S. Lany and A. Zunger, Phys. Rev. B: Condens. Matter Mater. Phys., 2008, 78, 235104.

46 C. Persson, Y.-J. Zhao, S. Lany and A. Zunger, Phys. Rev. B: Condens. Matter Mater. Phys., 2005, 72, 035211.

47 J. S. Park, S. Kim, Z. Xie and A. Walsh, Nat. Rev. Mater., 2018, 3, 194-210.

48 G. Kresse and J. Furthmüller, Comput. Mater. Sci., 1996, 6, 15-50.

49 G. Kresse and D. Joubert, Phys. Rev. B: Condens. Matter Mater. Phys., 1999, 59, 1758.

50 J. P. Perdew, K. Burke and M. Ernzerhof, Phys. Rev. Lett., 1996, 77, 3865.

51 J. Sun, A. Ruzsinszky and J. P. Perdew, Phys. Rev. Lett., 2015, 115, 036402.
52 J. Sun, R. C. Remsing, Y. Zhang, Z. Sun, A. Ruzsinszky, H. Peng, Z. Yang, A. Paul, U. Waghmare, X. Wu, et al., Nat. Chem., 2016, 8, 831.

53 J. Heyd, G. E. Scuseria and M. Ernzerhof, J. Chem. Phys., 2003, 118, 8207-8215.

54 D. Fritsch and S. Schorr, J. Phys.: Energy, 2020, 3, 015002.

55 J. Just, C. M. Sutter-Fella, D. Lützenkirchen-Hecht, R. Frahm, S. Schorr and T. Unold, Phys. Chem. Chem. Phys., 2016, 18, 15988-15994.

56 W. Wang, M. T. Winkler, O. Gunawan, T. Gokmen, T. K. Todorov, Y. Zhu and D. B. Mitzi, Adv. Energy Mater., 2013, 4, 1301465.

57 J. C. Slater, J. Chem. Phys., 1964, 41, 3199-3204.

58 S. Ma, H. Li, J. Hong, H. Wang, X. Lu, Y. Chen, L. Sun, F. Yue, J. W. Tomm, J. Chu, et al., J. Phys. Chem. Lett., 2019, 10, 79297936.

59 G. Rey, G. Larramona, S. Bourdais, C. Choné, B. Delatouche, A. Jacob, G. Dennler and S. Siebentritt, Sol. Energy Mater. Sol. Cells, 2018, 179, 142-151.

60 A. Alkauskas, Q. Yan and C. G. Van de Walle, Phys. Rev. B: Condens. Matter Mater. Phys., 2014, 90, 075202. 